\title{
The prevention and control of
} tuberculosis: an analysis based on a tuberculosis dynamic model derived from
the cases of Americans

Yan $\mathrm{Wu}^{1 \dagger}{ }^{1}$, Meng Huang ${ }^{1}$, Ximei Wang ${ }^{1}$, Yong $\mathrm{Li}^{1,2+^{*}}$ (D), Lei Jiang ${ }^{3}$ and Yuan Yuan ${ }^{4}$

\begin{abstract}
Background: Tuberculosis (TB), a preventable and curable disease, is claimed as the second largest number of fatalities, and there are 9,025 cases reported in the United States in 2018. Many researchers have done a lot of research and achieved remarkable results, but TB is still a severe problem for human beings. The study is a further exploration of the prevention and control of tuberculosis.

Methods: In the paper, we propose a new dynamic model to study the transmission dynamics of TB, and then use global differential evolution and local sequential quadratic programming (DESQP) optimization algorithm to estimate parameters of the model. Finally, we use Latin hypercube sampling (LHS) and partial rank correlation coefficients (PRCC) to analyze the influence of parameters on the basic reproduction number $\left(\boldsymbol{\mathcal { R }}_{0}\right)$ and the total infectious (including the diagnosed, undiagnosed and incomplete treatment infectious), respectively.

Results: According to the research, the basic reproduction number is computed as 2.3597 from 1984 to 2018, which means TB is also an epidemic in the US. The diagnosed rate is 0.6082 , which means the undiagnosed will be diagnosed after 1.6442 years. The diagnosed will recover after an average of 1.9912 years. Moreover, some diagnosed will end the treatment after 1.7550 years for some reason. From the study, it's shown that $2.40 \%$ of the recovered will be reactivated, and $13.88 \%$ of the newborn will be vaccinated. However, the immune system will be lost after about 19.6078 years.

Conclusion: Through the results of this study, we give some suggestions to help prevent and control the TB epidemic in the United States, such as prolonging the protection period of the vaccine by developing new and more effective vaccines to prevent TB; using the Chemoprophylaxis for incubation patients to prevent their conversion into active TB; raising people's awareness of the prevention and control of TB and treatment after illness; isolating the infected to reduce the spread of TB. According to the latest report in the announcement that came at the first WHO Global Ministerial Conference on Ending tuberculosis in the Sustainable Development Era, we predict that it is challenging to control TB by 2030.
\end{abstract}

Keywords: Tuberculosis (TB), Latin hypercube sampling (LHS), Partial rank correlation coefficients (PRCC), Basic reproduction number, Parameter estimation, Prevention and control measures

\footnotetext{
*Correspondence: yongli@yangtzeu.edu.cn

${ }^{\dagger}$ Yan Wu and Yong Li contributed equally to this work.

'School of Information and Mathematics, Yangtze University, Jingzhou

434023, China, Nanhuan Road, 434023 Jingzhou, China

${ }^{2}$ Institute of Applied Mathematics, Yangtze University, Nanhuan Road, 434023

Jingzhou, China

Full list of author information is available at the end of the article
}

(c) The Author(s). 2020 Open Access This article is licensed under a Creative Commons Attribution 4.0 International License, which permits use, sharing, adaptation, distribution and reproduction in any medium or format, as long as you give appropriate credit to the original author(s) and the source, provide a link to the Creative Commons licence, and indicate if changes were made. The images or other third party material in this article are included in the article's Creative Commons licence, unless indicated otherwise in a credit line to the material. If material is not included in the article's Creative Commons licence and your intended use is not permitted by statutory regulation or exceeds the permitted use, you will need to obtain permission directly from the copyright holder. To view a copy of this licence, visit http://creativecommons.org/licenses/by/4.0/. The Creative Commons Public Domain Dedication waiver (http://creativecommons.org/publicdomain/zero/1.0/) applies to the data made available in this article, unless otherwise stated in a credit line to the data. 


\section{Background}

To date, tuberculosis (TB) is regarded as one of the world's deadliest diseases caused by a single infectious agent, second only to acquired immunodeficiency syndrome (AIDS) caused by human immunodeficiency virus (HIV) $[1,2]$. TB is caused by Mycobacterium tuberculosis (MTB), which can be expelled into the air when the infected people cough, talk, sneeze or sing [3]. In most situations, MTB generally affects the lungs of infected individuals. Tuberculosis is so highly contagious that the susceptible are likely to develop TB even when they inhaled tiny particles containing MTB. The MTB is very small and can stay in the air for a long time and keep moving. The immune system is an essential defense mechanism that limits the growth and spread of MTB. If the immune system cannot suppress their growth, they will most likely spread throughout the body [4]. In fact, not all people infected with TB will be sick at once. Some people's incubation period may last from 1 year to several years. Nowadays, reliable TB tests do not exist [5], which causes many undiagnosed infectious and increases the difficulty in controlling TB.

It was reported in the Global Tuberculosis Report 2018 that there were 1.3 million deaths caused by TB [2]. People may be infected with TB in all countries and age groups, but overall, 90\% were adults (aged $\geq 15$ years), 9\% were people living with HIV (72\% in Africa). Most cases happened in following eight countries: India (27\%), China (9\%), Indonesia (8\%), the Philippines (6\%), Pakistan (5\%), Bangladesh (4\%), Nigeria (4\%), South Africa (3\%). WHO listed all of these countries and 22 other countries as the top 30 countries with the high burden of TB, which account for $87 \%$ of the world's cases. On the contrary, the global cases of the WHO European Region (3\%) and the WHO American Region (3\%) account for only 6\% [2].

Although the number of people infected with TB has been declining, there are about 10 to 20 thousands cases of TB every year in the last 20 years (see Table 1 ) [6], and the death rate is between 0.05 and 0.07 in America. Therefore, it is essential for researchers to explore the factors related to the infection, outbreak, and epidemic of TB so that we could take more measures to protect people from TB.

From 1945 to 1955, the widespread usage of antibiotics reduced TB mortality in the United States by $70 \%$, but the United States still has a severe TB epidemic [4, 8]. Based on decades of technology and experience, most active and latent $\mathrm{TB}$ can be effectively treated, and latent $\mathrm{TB}$ can be treated with Isoniazid. However, treatment can be effective only if the course of cures lasts no less than six months [4]. Active TB can be eliminated with a complex treatment regimen, coupled with the treatment of multiple drugs (Isoniazid, Rifampicin, Pyrazinamide) for nine months $[4,8]$.
In the United States, people who contract $\mathrm{TB}$ and receive treatment fall into two categories $[9,10]$. The first type of people are those who complete the treatment and eventually recover. The other type of the infected people do not complete treatment, due in part to lost data, patients' adverse reactions to the drug, or their refusal of treatment, etc. Another potential reasons that causes incomplete treatment is the high cost of TB treatment, which means a heavy burden to the general public. When the treatment is incomplete, the drug-resistant strains will reproduce, which may severely increase the difficulty of treatment $[4,11,12]$. According to the latest treatment outcome data for new cases in 2016, $82 \%$ of the people can successfully recover from TB. Apart from the reduction from 2015 to 2016 , there is another reduction from $86 \%$ in 2013 to $83 \%$ in 2015 [2].

Bacillus Calmette-Guerin (BCG) is a vaccine that has been used to prevent TB for a long time. The protection period of BCG vaccine usually varies from 10 to 20 years [13]. BCG precludes nearly 20 percent of children from getting infected, while the vaccine prevents approximately half of those already infected from getting worse [14]. The study of Fjallbrant et al. [15] suggested that the primary vaccination and revaccination of negative tuberculin skin test (TST) of young adults with BCG caused a significant increase in the T-helper 1 (Th1) response against mycobacterial antigens, suggesting a protective effect against TB. This gives support for the policy of primary vaccination as well as the revaccination under this setting and age group. In some areas where TB is prevalent, the primary BCG vaccination is essential, though should not be the primary measure for TB control. BCG is widely used in countries with a high burden of TB, but not those with a small burden. In the United States, BCG is often used by special people [16] but not widely used by the general public. As an alternative, Chemoprophylaxis $[17,18]$ is more affordable, easier to take, and can prevent latent infectious from turning into active TB. Thus, for some countries less inflicted by TB, Chemoprophylaxis is a better alternative to control TB [19].

On the dynamic model study, many researchers have devoted big efforts for the research of the epidemic law and transmission dynamics of TB. In 1962, Waaler et al. established the first dynamic model of TB based on a susceptible-infected-recovered (SIR) model [20]. From then on, many models that consider multiple influencing factors have been established, such as reinfection [21-23], vaccination [24-26], interactions with HIV [27, 28], reactivated [29,30], Chemoprophylaxis [19] and so on [31-34]. Revelle et al. considered prophylaxis, cure and BCG vaccination to research the optimal strategy to fight against TB, which was then extensively used to study the epidemic model of transmission for infectious disease in 1967 [35]. Buonomo et al. studied the global 


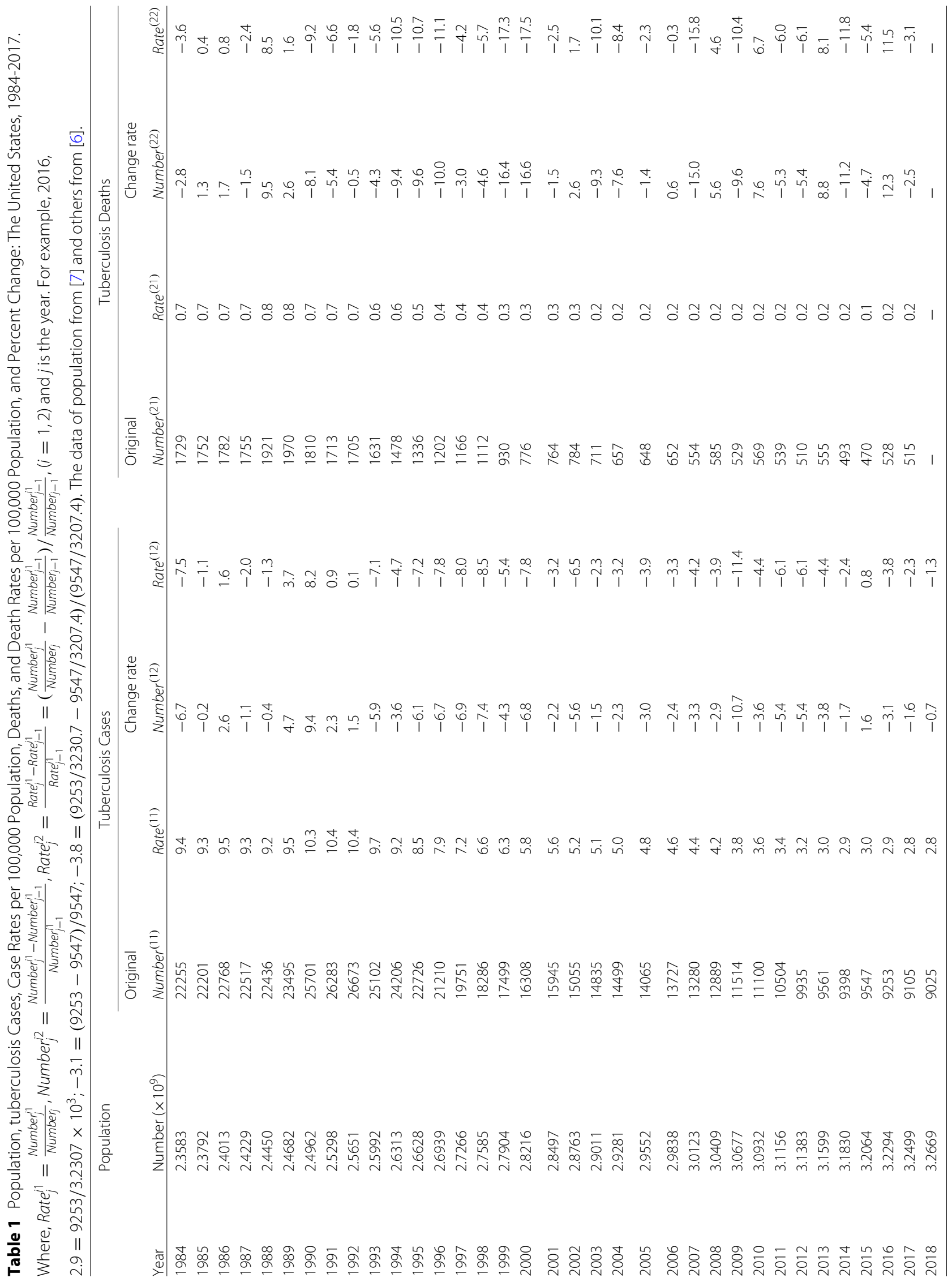


behavior of a non-linear susceptible-infectious-removed (SIR)-like epidemic model with a non-bilinear feedback mechanism [36]. The SEI model proposed by Bowong et al. exhibits the traditional threshold behavior [34]. Whang et al. used an SEIR model with the time-dependent parameters to develop a dynamic model for TB transmission in South Korea [37]. A mathematical model was proposed to understand the spread of TB disease in the human population for both pulmonary and drug-resistant subjects by Mishra et al. [38]. Three control factors must be considered simultaneously to decrease the threat of TB by Gao et al.: a preventive measure in the form of vaccination and two treatment measures aiming at the susceptible and individuals infected TB in the active stage and latent stage [39].

In developing countries, the increase of $\mathrm{TB}$ cases by a high level of undiagnosed infectious population and incompletely treated population is one of the greatest challenges to control TB. These people are more likely to develop multi-drug resistance relative to the diagnosed infectious population [21, 40]. According to the actual situation of TB in the United States, we considered several factors: slow-fast process [41-43], vaccination [2426], reinfection [21-23], reactivated [29, 30] and undiagnosed infection $[21,44]$. Then we referenced the modeling thought from D.P. Moualeu et al. [21, 45] and Liu et al. [24], and finally established our model. The biggest differences between our model and their model are the vaccination and recovery to the susceptible population $[21,24,45]$.

Our study aims to analyze the factors affecting TB based on the dynamics model, give several measures to control and prevent $\mathrm{TB}$, and predict the epidemic trend in America. The structure of this paper is as follows. In Section 2, we introduce our TB model expressed by ordinary differential equations (ODE) and define parameters. Then we describe the model assumptions and modeling ideas in detail and give the disease-free equilibrium and basic reproduction number. In Section 3, the model is simulated by global differential evolution and local sequential quadratic programming (DESQP) $[46,47]$ optimization algorithm based on the US cases. We analyze the fitting effect by the root mean square percentage error (RMSPE) and the mean absolute percentage error (MAPE). In Section 4, we make the uncertainty and sensitivity analysis of the parameters for our model by Latin hypercube sampling (LHS) and partial rank correlation coefficients (PRCC). We also analyze the sensitivity of each parameter on the basic reproduction number and the total infectious, respectively. In Section 5 and 6, we analyze the results, make some suggestions to prevent $\mathrm{TB}$, carry out simulation experiments, and then discuss the deficiency of our study. Finally, we summarize our research.

\section{Methods}

In this section, we introduce our new mathematical model, briefly explain the structure of our model, and then analyze the basic reproduction number.

\subsection{Model instruction}

The total population is denoted by $N(t)$, which is subdivided into the following seven sub-populations:

$V(t)$ vaccinated: healthy people vaccinated with $\mathrm{TB}$,

$S(t)$ susceptible: healthy people not exposed to TB,

$E(t)$ exposed: exposed to TB but not infectious,

$I(t)$ diagnosed infectious: infected with $\mathrm{TB}$ and diagnosed in hospital,

$J(t)$ undiagnosed infectious: infected with TB but undiagnosed in hospital,

$L(t)$ incompletely treated: have been diagnosed with active TB and begun their treatment in hospital or home, but quitted before the end,

$R(t)$ recovered: recovered from TB after treatment.

Considering slow-fast process [41-43], vaccination [2426], reinfection [21-23], re-activated [29, 30] and undiagnosed infection $[21,44]$ and referencing the modeling thought of the D.P. Moualeu et al. [21, 45] and Liu et al. [24], we display our dynamic model with a flow diagram shown in Fig. 1 and introduce the model in detail as the following:

For the dynamic system, there will be a recruitment to the dynamic system with an average scale $\Lambda$. Considering the vaccination, a proportion $\chi$ will be vaccinated (i.e., primary vaccination) and become the vaccinated class, and the remainder $(1-\chi)$ will not be vaccinated and become the susceptible class.

In fact, BCG will be ineffective for some newborns, and those newborns will be infected by contacting with the infectious, we assume the fraction as $\varepsilon$. The vaccine protection period takes 10 to 20 years [13], during which there will be a rate $\psi$ of losing the immune system and converging into the susceptible.

During the fast-slow process, the susceptible will be the exposed by contacting at a slow process $(1-p)$ and turning into the active TB at a fast $p$. Because the test of TB is not sensitive, there will be a fraction $f$ of diagnosed and $(1-f)$ undiagnosed. Here, we set $p_{1}=p f$ and $p_{2}=$ $p(1-f)$. We do not rule out that there will be a rate $\phi$, at which the susceptible will be revaccinated and become the vaccinated.

For the exposed, a proportion $r$ will use Chemoprophylaxis to prevent them from becoming active $\mathrm{TB}$, and the remainder $(1-r)$ will be the active TB at a rate $k$. For the active TB, there will be a fraction $h$ of individuals who will be diagnosed and healed, and thus $(1-h)$ undiagnosed.

In the diagnosed infectious, some will be recovered at a rate $g$. Some will be incompletely treated at a rate $\delta$. For the undiagnosed, some will become diagnosed 


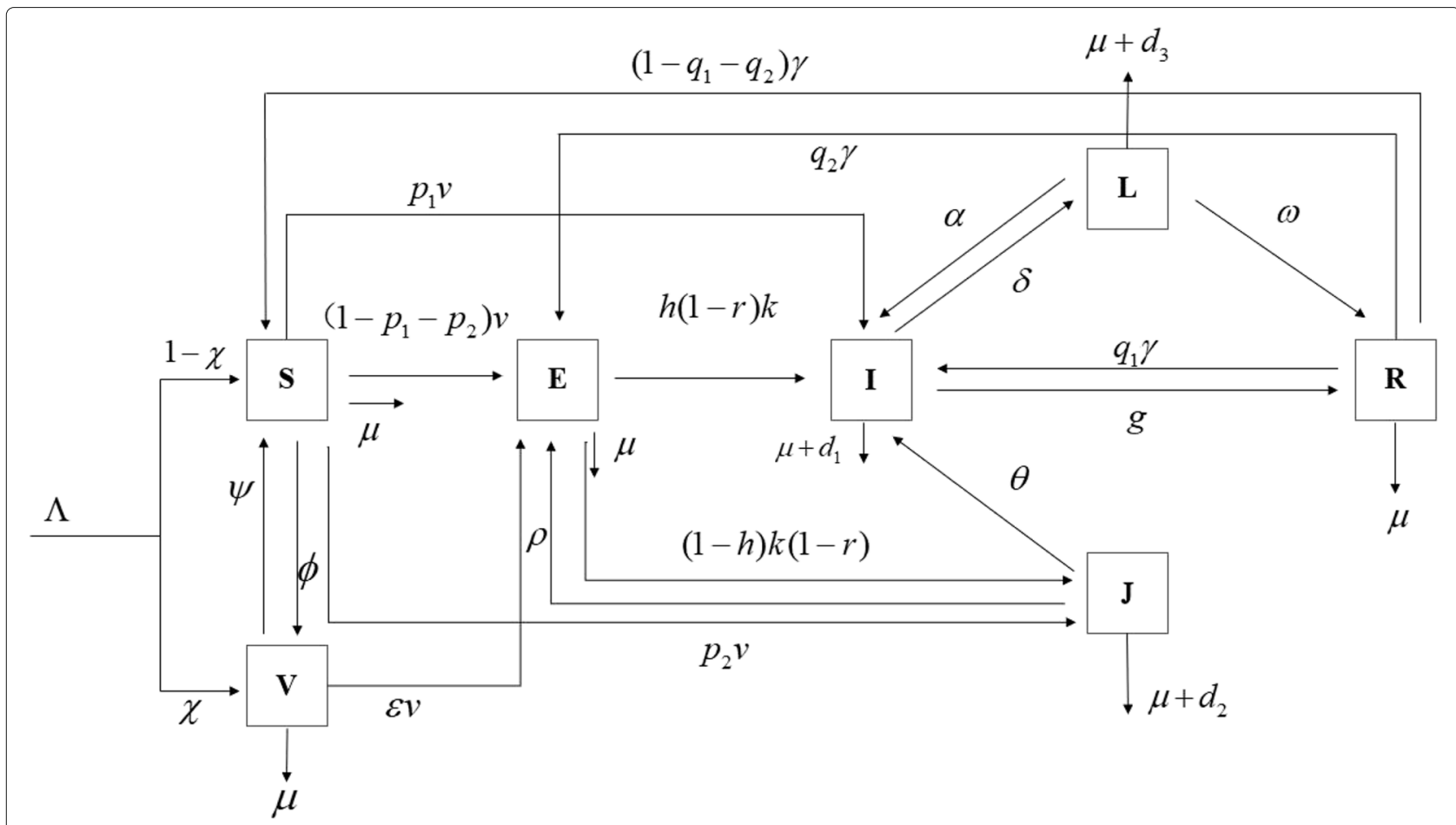

Fig. 1 The flow diagram for the compartment model of the transmission dynamics system of TB

and go to the hospital at a rate $\theta$; thanks to their immune system, some individuals will be the exposed at a rate $\rho$.

For the incomplete treatment, some will detect their illness and go to the hospital at a rate $\alpha$, while others will become recovered with the aid from self-immunity at a rate $\omega$.

Considering the re-infected and reactivated, the recovered will lose the immune system at a rate $\gamma$. A fraction $q_{1}$ of recovered people will be reactivated and become the diagnosed infectious; a fraction $q_{2}$ of the recovered will be re-infected but not be the active; and the remaining recovered individuals with a fraction $1-q_{1}-q_{2}$ will become the susceptible.

Lastly, the death of a certain number of people in each part of the system is resulted from natural causes. In our article, the main feature of our model is to consider the transmission route and mechanism of $\mathrm{TB}$ in the context of reality in a comprehensive way, which is of a great practical significance. Therefore, the conclusion will be closer to reality.

\subsection{Model introducing}

We give the definition and range of the parameters in Table 2, and proposed a mathematical model to state the transmission dynamics and epidemic of TB which is represented by the following system of ordinary differential equations:

$$
\left\{\begin{array}{l}
\frac{d V}{d t}=\Lambda \chi+\phi S-(\psi+\mu) V-\varepsilon v V \\
\frac{d S}{d t}=\Lambda(1-\chi)+\psi V+\left(1-q_{1}-q_{2}\right) \gamma R-\phi S-v S-\mu S, \\
\frac{d E}{d t}=\varepsilon v V+\left(1-p_{1}-p_{2}\right) v S+q_{2} \gamma R+\rho J-(1-r) k E-\mu E, \\
\frac{d I}{d t}=p_{1} v S+h(1-r) k E+\theta J+q_{1} \gamma R+\alpha L-(\delta+g) I-\left(\mu+d_{1}\right) I, \\
\frac{d J}{d t}=(1-h) k(1-r) E+p_{2} v S-(\theta+\rho) J-\left(\mu+d_{2}\right) J, \\
\frac{d L}{d t}=\delta I-(\alpha+\omega) L-\left(\mu+d_{3}\right) L, \\
\frac{d R}{d t}=g I+\omega L-(\gamma+\mu) R,
\end{array}\right.
$$

where

$$
N(t)=V(t)+S(t)+E(t)+I(t)+J(t)+L(t)+R(t) .
$$

The susceptible and the vaccinated are infected with TB through contacting individuals with active $\mathrm{TB}$ at a rate $v(I, J, L)$ given by:

$$
v=\frac{\beta_{1} I}{N}+\frac{\beta_{2} J}{N}+\frac{\beta_{3} L}{N},
$$

where $\beta_{i}, i=1,2,3$ are the rates that the diagnosed, undiagnosed infectious, and incompletely treated people sufficiently and effectively transmit TB to the susceptible or the vaccinated [1].

\subsection{Basic reproduction number}

The basic reproduction number $\mathcal{R}_{0}$ represents the number of infected during the initial patient's infectious (not sick) period [48]. Our model is a biological system model, so it must meet the biological conditions. Therefore, we only study the dynamic state of the solution of system (1) in the following feasible region: 
Table 2 The definition and value range of parameters for the model (1)

\begin{tabular}{|c|c|}
\hline Parameter & Definition \\
\hline$\mu$ & Natural mortality of human \\
\hline$\Lambda$ & New individuals coming into the system \\
\hline$\alpha$ in $[0.1,0.4]$ & $\begin{array}{l}\text { The rate for incompletely treated going to diagnosed } \\
\text { infectious }\end{array}$ \\
\hline$\beta_{1}$ in $[2,6]$ & The transmission rate of diagnosed infectious \\
\hline$\beta_{2}$ in $[0.1,0.4]$ & The transmission rate of undiagnosed infectious \\
\hline$\beta_{3}$ in $[4,6]$ & The transmission rate of incompletely treated \\
\hline$\chi$ in $[0.08,0.3]$ & The natural vaccination ratio of the newborn babies \\
\hline$d_{1}$ in $[0.05,0.1]$ & TB-related mortality of diagnosed infectious \\
\hline$d_{2}$ in $[0.5,0.8]$ & TB-related mortality of undiagnosed infectious \\
\hline$d_{3}$ in $[0.09,0.3]$ & TB-related mortality of incompletely treated \\
\hline$\delta$ in $[0.5,0.8]$ & $\begin{array}{l}\text { Rate for diagnosed infectious coming into the } \\
\text { incompletely treated }\end{array}$ \\
\hline$\varepsilon$ in $[0,0.3]$ & $\begin{array}{l}\text { Indicate the reduction in risk of infection due to } \\
\text { vaccination }\end{array}$ \\
\hline$g$ in $[0.4,0.8]$ & Recovery rate of the diagnosed infectious \\
\hline$\gamma$ in $[0.125,0.6]$ & Reactivated rate of recovered individuals \\
\hline$h$ in $[0.2,0.6]$ & Detection ratio of active $\mathrm{TB}$ \\
\hline$k$ in $[0.033,0.333]$ & Rate of progression to infectious \\
\hline$p_{1}$ in $[0.02,0.08]$ & $\begin{array}{l}\text { The proportion of susceptible individuals who } \\
\text { become diagnosed infectious }\end{array}$ \\
\hline$p_{2}$ in $[0,0.5]$ & $\begin{array}{l}\text { The proportion of susceptible individuals who } \\
\text { become undiagnosed infectious }\end{array}$ \\
\hline$\psi$ in $[0.05,0.2]$ & Loss of vaccination rate \\
\hline$\phi$ in $[0.05,0.1]$ & Vaccine coverage rate \\
\hline$q_{1}$ in $[0,0.4]$ & The ratio of recovered relapsing into the infectious \\
\hline $9_{2}$ in $[0.15,0.6]$ & The ratio of recovered reinfected into the exposure \\
\hline$r$ in $[0.7,0.9]$ & Chemoprophylaxis of the exposed \\
\hline$\rho$ in $[0.15,0.25]$ & $\begin{array}{l}\text { Rate of progression from undiagnosed infectious to } \\
\text { exposed }\end{array}$ \\
\hline$\theta$ in $[0.6,0.9]$ & $\begin{array}{l}\text { Rate of progression from undiagnosed infectious to } \\
\text { diagnosed infectious }\end{array}$ \\
\hline$\omega$ in $[0.15,0.25]$ & Recovery rate of the incompletely treated \\
\hline
\end{tabular}

$$
\Omega=\left\{(V, S, E, I, J, L, R) \in \mathbb{R}_{+}^{7}: V+S+E+I+J+L+R \leq \frac{\Lambda}{\mu}\right\},
$$

which can be confirmed as positively invariant. Therefore, we restrict our attention to the dynamics of the model (1) in $\Omega$.

For the threshold system, when $\mathcal{R}_{0}<1$, the model will stabilize to the disease-free equilibrium, and the disease will be controlled and eventually become extinct. When $\mathcal{R}_{0}>1$, the model will stabilize to the endemic equilibrium, and the disease will develop into an endemic disease. For other complex systems, this conclusion may not be valid, for example, backward bifurcation, multistable system and other complex dynamic behaviors may occur. Therefore, the smaller $\mathcal{R}_{0}$ is, the easier to control TB $[49,50]$. Here, we use the next-generation matrix approach to calculate the basic reproduction number $\mathcal{R}_{0}$, which was proposed by Van den Driessche, etc [49]. The detail calculation is given in the Appendix.

\section{Simulation}

Based on the reported data from 1984 to 2018 by WHO [6] and the model (1), a global differential evolution and local sequential quadratic programming (DESQP) optimization algorithm was conducted to estimate the undetermined parameters $[47,51]$. DESQP, which combines differential evolution (DE) [46] and local sequential quadratic programming (SQP) $[52,53]$, is a method used to search for the optimal solution of DE. In the method, DE is used as a base level search and SQP is used as a local search. DE is first applied to the short term of the problem to find the best solution. This optimal solution is given to SQP as an initial condition to fine tune the solution to reach the global optimum or near global optimum.

We get the estimated value, standard deviation, confidence interval, P-value and t-statistic of the parameters, as listed in Table 3. Based on the estimated results, the basic reproduction number $\mathcal{R}_{0}$ can be calculated $\mathcal{R}_{0}=$ 2.3597. Aandahl et al. [54] in 2014 specified an informative in [55] and improved the convergence performance of the Markov chain Monte Carlo (MCMC) sampler in [56], then estimated the reproduction number as: 2.1 (95\% CI: 1.542.66) for the approximate method in [55] and 2.05 (95\% CI: 1.55-2.63) for the exact method in [56].

The real data and the model results are shown in the following Fig. 2. We evaluate the fitting effect of our established model through the root mean square percentage error (RMSPE) and the mean absolute percentage error (MAPE) which are significant evaluation indicators. The RMSPE and the MAPE are defined as:

$$
\begin{aligned}
& \text { MAPE }=\left(\frac{1}{n} \sum_{t=1}^{n}\left|\frac{I(t)^{*}-I(t)}{I(t)^{*}}\right|\right) \times 100 \%, \\
& \text { RMSPE }=\sqrt{\frac{\sum_{t=1}^{n}\left[\left(I(t)^{*}-I(t)\right) / I(t)^{*}\right]^{2}}{n-1}} \times 100 \%,
\end{aligned}
$$

where $I(t)^{*}$ is the real value at time $t, I(t)$ is its fitting value, and $n$ is the number of data used for prediction. The criteria for MAPE and RMSPE are shown in Table 4 $[57,58]$. We use model (1) to simulate the number of the infected, where $\mathrm{MAPE}=4.7245 \%$ and $\mathrm{RMSPE}=5.7676 \%$, which means the fitting effect is very desirable and our system has strong prediction ability and high prediction accuracy. 
Table 3 The t-statistic, P-value, Cl Bound, Standard deviation, the estimated value of parameters, and the initial condition of each compartment of the model (1)

\begin{tabular}{|c|c|c|c|c|c|c|}
\hline $\begin{array}{l}\text { Parameter } \\
\text { initial value }\end{array}$ & Value & $\begin{array}{l}\text { Standard } \\
\text { deviation }\end{array}$ & $\begin{array}{l}\text { CI Low } \\
\text { Bound }\end{array}$ & $\begin{array}{l}\mathrm{Cl} \text { High } \\
\text { Bound }\end{array}$ & P-Value & t-statistic \\
\hline$\alpha$ & 0.1002 & 0.0340 & -0.0081 & 0.2085 & 0.0603 & 2.9434 \\
\hline$\beta_{1}$ & 4.4381 & 0.2390 & 3.6774 & 5.1987 & 0.0003 & 18.5687 \\
\hline$\beta_{2}$ & 0.2994 & 0.0312 & 0.2002 & 0.3985 & 0.0024 & 9.6079 \\
\hline$\beta_{3}$ & 5.8662 & 0.1946 & 5.2469 & 6.4855 & 0.0001 & 30.1466 \\
\hline$\chi$ & 0.1388 & 0.0425 & 0.0036 & 0.2740 & 0.0469 & 3.2664 \\
\hline$d_{1}$ & 0.0506 & 0.0240 & -0.0256 & 0.1269 & 0.1251 & 2.1126 \\
\hline$d_{2}$ & 0.7257 & 0.1033 & 0.3968 & 1.0546 & 0.0059 & 7.0222 \\
\hline$d_{3}$ & 0.0947 & 0.0572 & -0.0875 & 0.2769 & 0.1966 & 1.6543 \\
\hline$\delta$ & 0.5698 & 0.1622 & 0.0535 & 1.0861 & 0.0391 & 3.5121 \\
\hline$\varepsilon$ & 0.5539 & 0.1130 & 0.1944 & 0.9134 & 0.0162 & 4.9029 \\
\hline$g$ & 0.5022 & 0.1049 & 0.1682 & 0.8362 & 0.0173 & 4.7856 \\
\hline$\gamma$ & 0.1444 & 0.0388 & 0.0210 & 0.2679 & 0.0337 & 3.7225 \\
\hline$h$ & 0.3998 & 0.0687 & 0.1811 & 0.6184 & 0.0101 & 5.8184 \\
\hline k & 0.0421 & 0.0154 & -0.007 & 0.0913 & 0.0721 & 2.7281 \\
\hline$\Lambda$ & $3.3510 \times 10^{3}$ & 6.6920 & $3.3300 \times 10^{3}$ & $3.3723 \times 10^{3}$ & $1.7562 \times 10^{-8}$ & $5.0076 \times 10^{2}$ \\
\hline$p_{1}$ & 0.025 & 0.0125 & -0.0146 & 0.0647 & 0.1384 & 2.0072 \\
\hline$p_{2}$ & 0.3414 & 0.0538 & 0.1702 & 0.5125 & 0.0079 & 6.3472 \\
\hline$\phi$ & 0.0500 & 0.0255 & -0.0311 & 0.1311 & 0.1444 & 1.9630 \\
\hline$\psi$ & 0.0510 & 0.0123 & 0.0120 & 0.0901 & 0.0252 & 4.1617 \\
\hline$q_{1}$ & 0.0240 & 0.0077 & $6.7543 \times 10^{-4}$ & 0.0486 & 0.0535 & 3.0952 \\
\hline$q_{2}$ & 0.5425 & 0.0390 & 0.4183 & 0.6667 & 0.0008 & 13.9023 \\
\hline$r$ & 0.9219 & 0.0259 & 0.8394 & 1.0045 & 0.0000 & 35.5518 \\
\hline$\rho$ & 0.1993 & 0.0769 & -0.0456 & 0.4442 & 0.0811 & 2.5903 \\
\hline$\theta$ & 0.6082 & 0.1642 & 0.0856 & 1.1308 & 0.0342 & 3.7037 \\
\hline$\omega$ & 0.1986 & 0.0233 & 0.1245 & 0.2726 & 0.0034 & 8.5350 \\
\hline$V(0)$ & $9.2311 \times 10^{5}$ & 172.1804 & $9.2256 \times 10^{5}$ & $9.2366 \times 10^{5}$ & $1.4311 \times 10^{-11}$ & $5.3613 \times 10^{3}$ \\
\hline$s(0)$ & $4.6139 \times 10^{6}$ & 101.8849 & $4.6136 \times 10^{6}$ & $4.6142 \times 10^{6}$ & $2.3746 \times 10^{-14}$ & $4.5286 \times 10^{4}$ \\
\hline$E(0)$ & $1.1199 \times 10^{6}$ & 164.3129 & $1.1194 \times 10^{6}$ & $1.1204 \times 10^{6}$ & $6.9652 \times 10^{-12}$ & $6.8157 \times 10^{3}$ \\
\hline I(0) & $2.2255 \times 10^{4}$ & - & - & - & - & - \\
\hline$J(0)$ & $4.8511 \times 10^{4}$ & 21.2908 & $4.8443 \times 10^{4}$ & $4.8579 \times 10^{4}$ & $1.8643 \times 10^{-10}$ & $2.2785 \times 10^{3}$ \\
\hline$L(0)$ & $5.7244 \times 10^{2}$ & 2.5804 & $5.6423 \times 10^{2}$ & $5.8065 \times 10^{2}$ & $2.0199 \times 10^{-7}$ & $2.2184 \times 10^{2}$ \\
\hline$R(0)$ & $6.9875 \times 10^{2}$ & 1.9114 & $6.9267 \times 10^{2}$ & $7.0483 \times 10^{2}$ & $4.5139 \times 10^{-8}$ & $3.6557 \times 10^{2}$ \\
\hline
\end{tabular}

\section{Sensitivity analysis}

In model (1), the precise estimation of parameter values is one of the greatest challenges. Direct measurements of specific biological parameters are rare, and many parameters are estimated within a broad range of values identified by fitting the model with limited experimental data [59]. Therefore, the estimation of the parameters is always associated with uncertainty analysis (UA) and sensitivity analysis (SA). It is vital to study the influence of the uncertainty of these parameters on the model, as this will not only help us successfully use the mathematical and computational models of biological systems as prediction tools, but also comprehend the functions of biological systems.

Some factors are which we cannot control in the model (1). Generally, researchers would choose the factors that we can control to analyze the sensitivity so that we can find effective measures to eliminate TB. Since we cannot control the death rate $d_{1}, d_{2}$, and $d_{3}$ in our analysis, we only did sensitivity analysis for 22 parameters that we can control in an attempt to eliminate TB. To find how the 


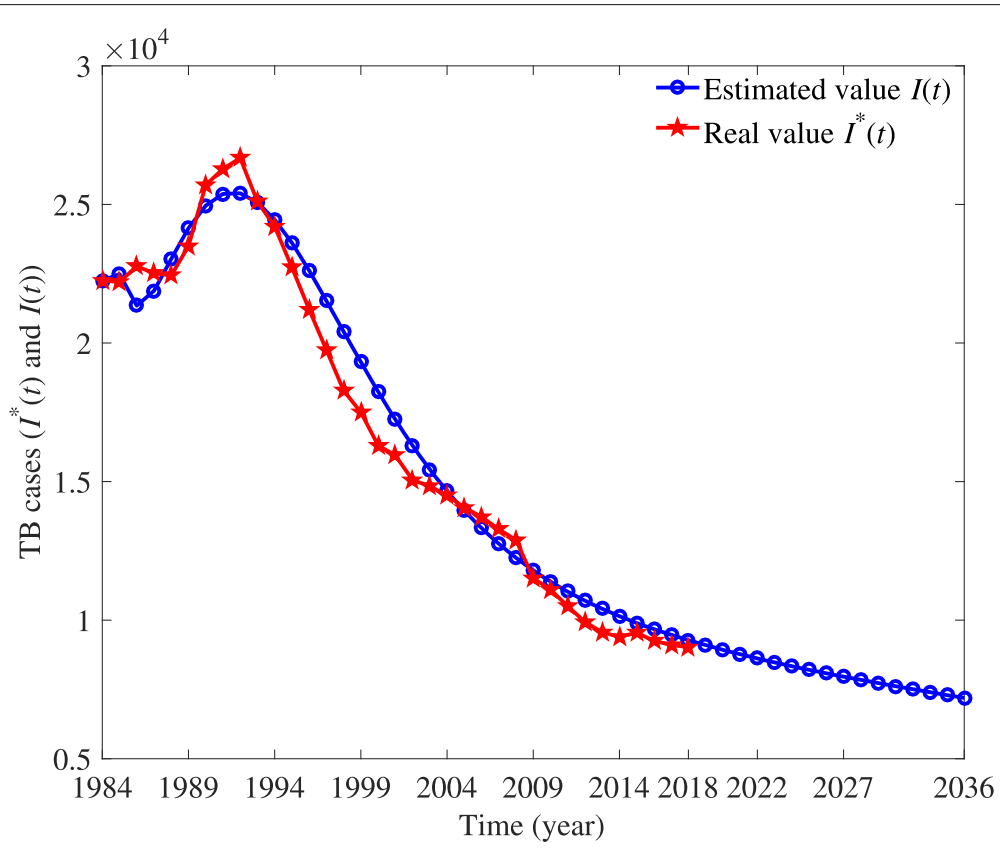

Fig. 2 The comparison of real data and fitted data and the projection for the future status of TB

parameters impact on the outcome, a general and better way is to do the sensitivity analysis for each parameter. The ideal method is to use Latin Hypercube Sampling (LHS) and Partial Rank Correlation Coefficient (PRCC) to study the dependence of model parameters on the basic reproduction number and total infectious $[60,61]$.

\subsection{Latin hypercube sampling (LHS)}

Generally, the input factors of the most mathematical and computational model consist of initial conditions and parameters, which are independent and dependent model variables. Thanks to natural variation, lack of current techniques, measurement error, etc., the parameters are not always known with adequate certainty [61]. The purpose of uncertainty analysis (UA) is to solve these problems. UA can quantify the degree of confidence in the experimental data and the estimated values of the parameters [61].

In the article, introduced by Mckay et al., the most popular and efficient Latin hypercube sampling-LHS that belongs to Monte Carlo (MC) class of sampling methods was used to perform UA [61]. MC method, a common

Table 4 The criteria for MAPE and RMSPE

\begin{tabular}{ll}
\hline MAPE and RMSPE & Forecasting power \\
\hline$<10 \%$ & Highly accurate forecasting \\
$10-20 \%$ & Good forecasting \\
$20-50 \%$ & Reasonable forecasting \\
$>50 \%$ & Inaccurate forecasting \\
\hline
\end{tabular}

algorithm to solve various computational problems, can evaluate multiple models, the results of which can not only be used to perform SA, but also to determine the uncertainty of model inputs. LHS can unbiasedly estimate the average output of the model, and fewer samples are required to achieve the same accuracy as simple random sampling [61].

The remaining 22 parameters have been chosen to do uncertainty analysis. We assume each parameter to be a random variable with normal distribution to analyze the uncertainty in the value of these parameters. Normal distribution for all parameters with the mean (i.e., estimated value) and variance value (i.e., square of standard deviation) are given in Table 3. Latin hypercube sampling has been used to sample for these parameters considered for the sensitivity analysis. Here, we set the sample size $\mathrm{N}=2000$. Using Latin hypercube sampling method and probability density function for each parameter is stratified into 2000 equiprobable $(1 / 2000)$ serial intervals. Then a single value is randomly chosen from each interval. This produces 2000 sets of values for each parameter, and we can compute 2000 sets of values for $\mathcal{R}_{0}$ from 2000 sets of different parameter values and get the distribution hist of $\mathcal{R}_{0}$, as is shown in Fig. 3.

\subsection{Partial rank correlation coefficient (PRCC)}

Sensitivity analysis (SA) is a quantitative way to analyze the effects of the parameter uncertainty on the model's outputs. Based on the parameters, we are able to raise presumptions about the biological system that actuates the system behavior, which can be measured by conducting 


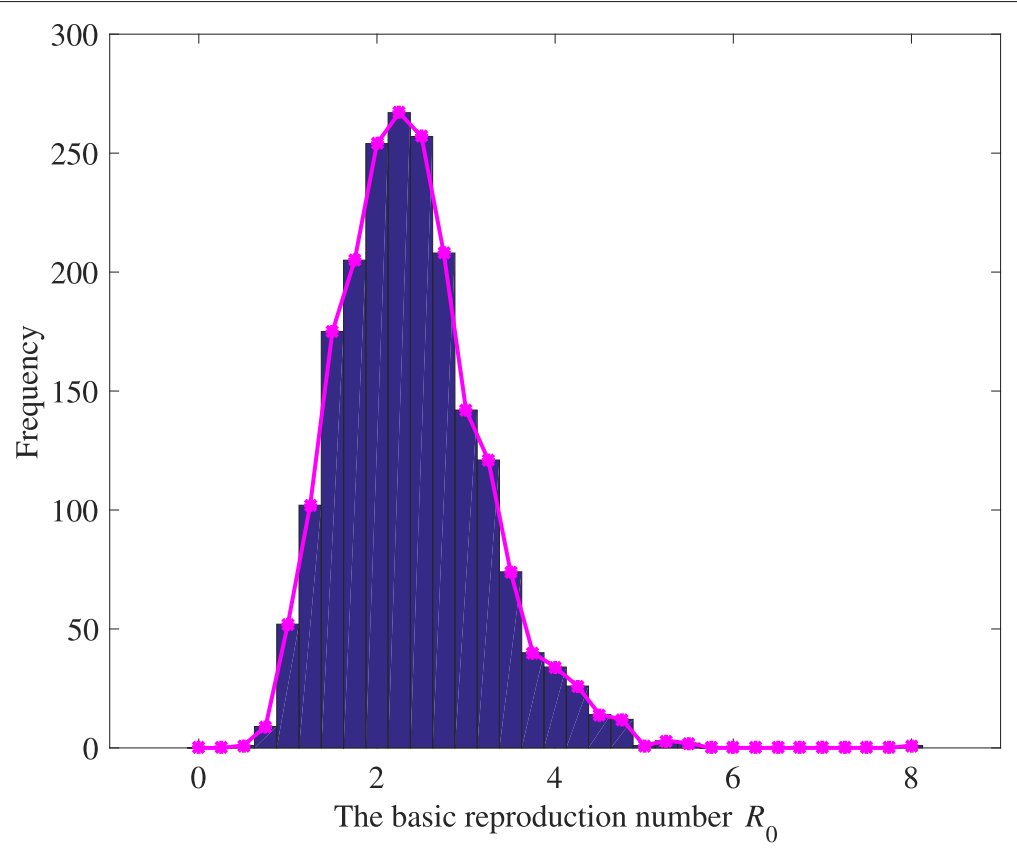

Fig. 3 The distribution of the basic reproduction number $\boldsymbol{\mathcal { R }}_{0}$

experiments [62]. Local SA techniques, one class of SA, investigate on the effects of small variations in individual parameters around some nominal point and have been applied to a number of signal transduction and metabolic pathway models [63, 64]. Since the most influential parameters are determined, the predictability of the model can be significantly enhanced.

In this section, we compute PRCC to analyze the sensitivity of the parameters to the $\mathcal{R}_{0}$ and the total infectious so as to identify the parameters that have great effect on the variability in the outcome and how those parameters affect both $\mathcal{R}_{0}$ and the total infectious. Here we compute the PRCC of $\mathcal{R}_{0}$ and the total infectious based on the LHS matrix, the result of which can be seen from Fig. 4, Table 5. In our experiment, we assume that the parameters have a significant effect when $\mathrm{P}$-value $<0.01$.

From Fig. 4, we can easily see the different parameters have different extent of the effect on both $\mathcal{R}_{0}$ and the total infectious, so it is complex to take proper measures to control TB. To better control TB, we emphasize on analyzing the parameters whose PRCC $>0.2$. Apart from that, we assume that these parameters have a high degree and a significant effect on $\mathcal{R}_{0}$ and the total infectious. Expect for the uncontrollable factor (which we cannot take relative measure to control TB) from Fig. 4, we can easily see that $r, \omega, g$ and $\phi$ have significantly positive affect on both the $\mathcal{R}_{0}$ and the total infectious, while $\varepsilon, k, \psi, \delta$ and $\theta$ have significantly negative effect on both the $\mathcal{R}_{0}$ and the total infectious.

\section{Results}

In this section, we present the results of the simulation for the model. In general, parameter estimation is an iterative process, in which we use the current parameter values as the initial values of the next iteration [1]. All the parameter values of the first iterative process are set to be their initial guess values, which are estimated with the lowest sub-condition. Then parameters estimation is carried out with a limited list of previously non-identifiable parameters. Finally, we repeat the estimation process and check all the estimated parameters to see whether the new values of the previously unrecognized parameters affect the values of the identifiable parameters. We use the data of TB cases (i.e., diagnosed infectious) in America from 1984 to 2018 (see Table 1) published by the Centers for Disease Control and Prevention (CDC) to estimate the parameters of the model (1).

In our model, some parameters have been estimated by WHO, some evaluated by the TB researchers, and the others remain uncertain. We specify some parameter values as listed below.

(1) The natural mortality $\mu$ : It is assumed to be equal to the inverse of the life expectancy at birth and $1 / \mu=79.30$ is the average human lifespan. Accordingly $\mu=0.0126$ [65].

(2) Progress rate of the exposed to infectious individuals (including diagnosed and undiagnosed infectious) $k$ : Based on the parameter estimation $k=0.0421$, the incubation period of TB is $1 / k=23.7529$ years. The latent TB infection lasts from 1 year to several years in general $[1,19,66]$. 


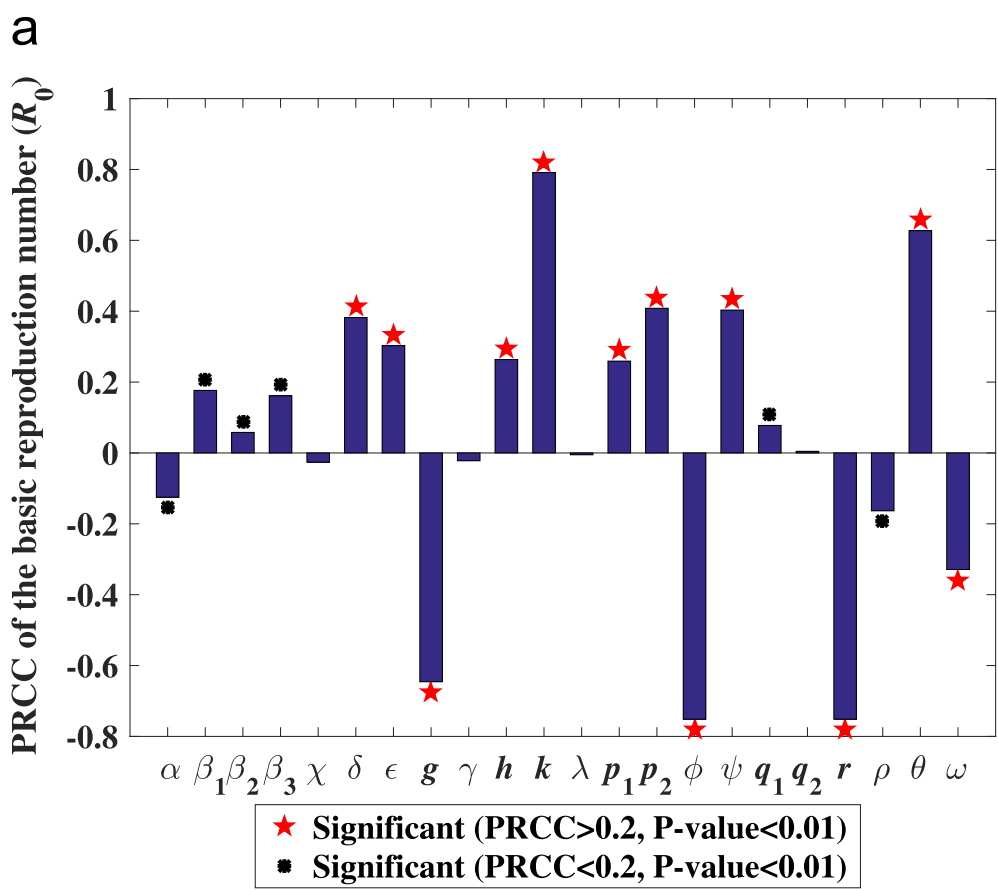

Parameter

b

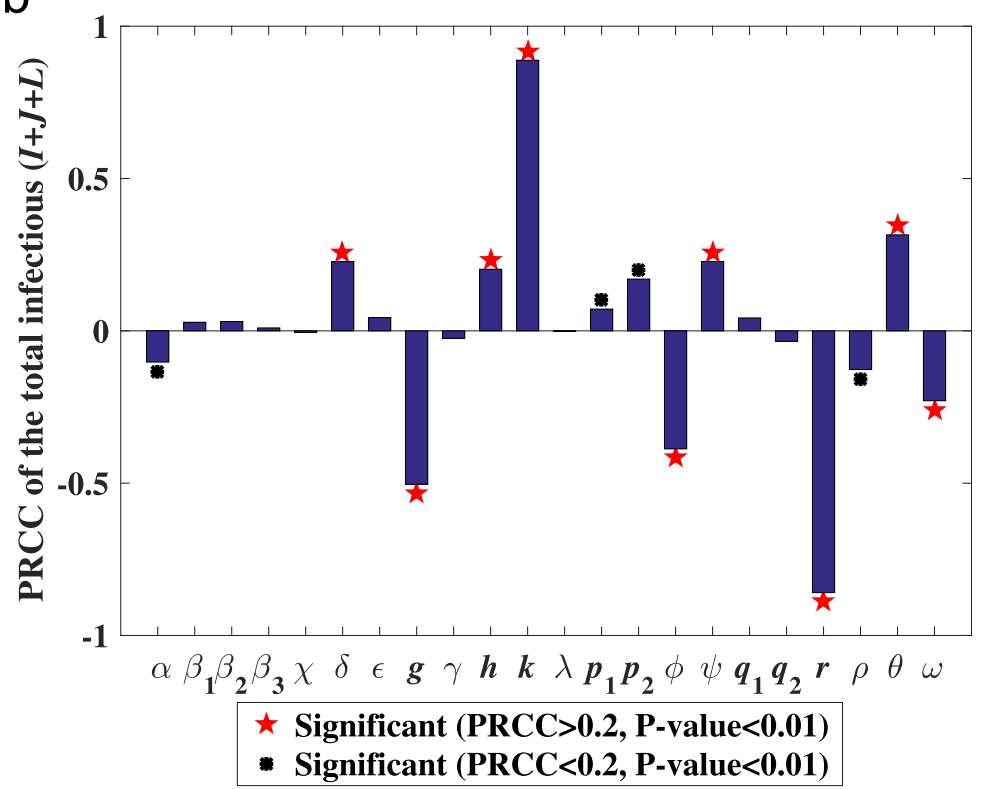

Parameter

Fig. 4 (a) show the PRCC of parameters with $\boldsymbol{\mathcal { R }}_{0}$; (b) show the PRCC of parameters with the total infected. Here,we assume that when P-value $<0.01$, the parameters have significant effect $\boldsymbol{\mathcal { R }}_{0}$ and the total infectious. To better control TB, we emphasize on analyzing the parameters whose PRCC $>0.2$

(3) Recovery rate of the diagnosed infectious $g$ : In our simulation, $g=0.5022$, and the course of recovery for the diagnosed infection is estimated $1 / g=1.9912$ years. By 2010, the course of treatment of the first-time TB patient is normally treated in 6 months; and the course of treatment of the reinfected tuberculosis patient is usually 18-24 months $[67,68]$.

(4) Diagnosis rate $\theta: \theta=0.6082$ per year, which means the undiagnosed individual will be diagnosed with active $\mathrm{TB}$ after $1 / \theta=1.6442$ years. Generally, 
Table 5 The value of PRCC between each parameter and $\boldsymbol{\mathcal { R }}_{0}$ and the total infectious

\begin{tabular}{|c|c|c|c|c|}
\hline \multirow{2}{*}{ Parameters } & \multicolumn{2}{|r|}{$\boldsymbol{\mathcal { R }}_{0}$} & \multicolumn{2}{|c|}{ Total infectious } \\
\hline & PRCC & P-value & PRCC & P-value \\
\hline$\alpha$ & -0.1025 & $4.8981 \times 10^{-6}$ & -0.1248 & $2.5216 \times 10^{-8}$ \\
\hline$\beta_{1}$ & 0.0281 & $2.1229 \times 10^{-1}$ & 0.1756 & $2.5743 \times 10^{-15}$ \\
\hline$\beta_{2}$ & 0.0306 & $1.7314 \times 10^{-1}$ & 0.0580 & $9.9135 \times 10^{-3}$ \\
\hline$\beta_{3}$ & 0.0096 & $6.7013 \times 10^{-1}$ & 0.1614 & $5.1136 \times 10^{-13}$ \\
\hline$\chi$ & -0.0050 & $8.2461 \times 10^{-1}$ & -0.0259 & $2.5024 \times 10^{-1}$ \\
\hline$\delta$ & 0.2269 & $1.5761 \times 10^{-24}$ & 0.3822 & $7.6609 \times 10^{-70}$ \\
\hline$\varepsilon$ & 0.0437 & $5.2022 \times 10^{-2}$ & 0.3028 & $3.0568 \times 10^{-43}$ \\
\hline$g$ & -0.5037 & $7.7684 \times 10^{-128}$ & -0.6457 & $6.9717 \times 10^{-234}$ \\
\hline$\gamma$ & -0.0247 & $2.7261 \times 10^{-1}$ & -0.0220 & $3.2766 \times 10^{-1}$ \\
\hline$h$ & 0.2023 & $1.0012 \times 10^{-19}$ & 0.2636 & $8.0884 \times 10^{-33}$ \\
\hline k & 0.8883 & 0.0000 & 0.7931 & 0.0000 \\
\hline$\Lambda$ & -0.0015 & $9.4530 \times 10^{-1}$ & -0.0051 & $8.2052 \times 10^{-1}$ \\
\hline$p_{1}$ & 0.0713 & $1.4980 \times 10^{-3}$ & 0.2591 & $9.9110 \times 10^{-32}$ \\
\hline$p_{2}$ & 0.1699 & $2.7490 \times 10^{-14}$ & 0.4083 & $2.3152 \times 10^{-80}$ \\
\hline$\phi$ & -0.3874 & $6.9735 \times 10^{-72}$ & -0.7513 & 0.0000 \\
\hline$\psi$ & 0.2276 & $1.1225 \times 10^{-24}$ & 0.4032 & $3.1138 \times 10^{-78}$ \\
\hline$q_{1}$ & 0.0423 & $5.9895 \times 10^{-2}$ & 0.0776 & $5.4967 \times 10^{-4}$ \\
\hline$q_{2}$ & -0.0341 & $1.2968 \times 10^{-1}$ & 0.0046 & $8.3880 \times 10^{-1}$ \\
\hline$r$ & -0.8593 & 0.0000 & -0.7511 & 0.0000 \\
\hline$\rho$ & -0.1269 & $1.4744 \times 10^{-8}$ & -0.1633 & $2.6748 \times 10^{-13}$ \\
\hline$\theta$ & 0.3151 & $7.2127 \times 10^{-47}$ & 0.6273 & $5.8105 \times 10^{-217}$ \\
\hline$\omega$ & -0.2294 & $4.7563 \times 10^{-25}$ & -0.3289 & $3.9428 \times 10^{-51}$ \\
\hline
\end{tabular}

some people with active TB are difficult to be diagnosed.

(5) Progression rate at which diagnosed infectious people become incompletely treated $\delta$ : It has been estimated as $\delta=0.5698$ per years, which means the diagnosed people may give up treatment after $1 / \delta=1.7550$ years. Generally, the average convalescence period of TB is around 1 year, which means after treated for 1 year, people will consider themselves to be recovered, but this is not the case [49].

(6) Reactivated ratio $q_{1}$ and reinfected ratio $q_{2}$ : It has been estimated as $q_{1}=2.40 \%, q_{2}=54.25 \%$. This shows that relapse for most people is a slow progress, but reinfected is fast. $\left(1-q_{1}-q_{2}\right)=43.35 \%$, which means the people will lose the immune system and become the susceptible.

(7) Progress rate at which incompletely treated people become diagnosed infectious $\alpha$ : It has been estimated as $\alpha=0.1002$, which means that the patients that are incompletely treated may be retreated after $1 / \alpha=9.9800$ years.
(8) The natural vaccination rate of the newborn babies $\chi$ : It has been estimated as $\chi=13.88 \%$. In America, only a few people get BCG vaccination [16-18]. The United States and other western countries with low TB burden do not necessarily require people to be vaccinated against BCG for newborns. Some Americans will be vaccinated per doctor's advice [16-18].

(9) Progress rate $\psi$ : the rate at which the vaccinated become the susceptible. It has been estimated as $\psi=$ 0.0510 which means the vaccination may be invalid after $1 / \psi=19.6078$ years. BCG vaccine duration varies widely, ranging from 10 to 20 years [13].

(10) Progress rate $\gamma$ : the rate at which the recovered individuals lose the immune system. It is estimated as $\gamma=$ 0.1444 per year which means the recovered individuals may lose the immune system after $1 / \gamma=6.9252$ years.

(11) Progression rate at which the undiagnosed become the exposed $\rho$. It has been estimated as $\rho=0.1993$ which means it takes $1 / \rho=5.0176$ years or so for the undiagnosed to become non-infectious individuals (exposed).

(12) Detection rate of active TB $h$ : It is estimated as $h=$ $39.98 \%$. This shows that $60.02 \%$ of tuberculosis patients will not be diagnosed or will not be diagnosed for a short time.

(13) Vaccination coverage $\phi$ : It has been estimated as $\phi=0.0500$, which means after an average of $1 / \phi=$ 20.0000 years, people will lose antibodies to TB and be vaccinated again. Generally, BCG is not widely used in America, and the adults also will not choose to be vaccinated if it's unnecessary $[16,17]$.

(14) Chemoprophylaxis rate $r$ : It has been estimated as $r=0.9219$. Dye et al. have estimated $r=0.7[19,69,70]$.

(15) Recovery rate of the incompletely treated $\omega$ : It has been estimated as $\omega=0.1986$, which means some incompletely treated individuals will naturally recover after $1 / \omega=5.0352$ years. Bacaër et al. have estimated that the natural recovery from HIV-negative TB and HIV-positive TB takes 0.1390 and 0.2400 per year, respectively. HIVnegative TB and HIV-positive TB will be recovered after 7.1900 years and 4.1700 years without treatment [71].

(16) The rate of the susceptible become the diagnosed and undiagnosed infectious $p_{1}, p_{2}$ : It is estimated that $p_{1}=2.5 \%$ and $p_{2}=34.14 \%$, which means $2.5 \%+$ $34.14 \%=36.54 \%$ of the people will be sick at once after infected by TB, while $2.5 \%$ have severe symptoms and $34.14 \%$ have mild symptoms and do not get a diagnosis. The remaining $\left(1-p_{1}-p_{2}\right)=63.46 \%$ of people are those who proceed to a slow progression of TB infection become the exposed.

\section{Discussion}

In the section, we discuss the sensitivity of parameters to the $\mathcal{R}_{0}$ and the total infectious. Furthermore, we suggest several measures to prevent TB. TB is a prevalent 
infectious disease in the world and the infectious are spread worldwide. It is vital to seize the leading causes and find the best measures to prevent and control the disease. In the article, we construct a TB model to study the transmission dynamic and provide some measures to control and prevent TB in the US. To find more ways to prevent $\mathrm{TB}$, we analyze many factors that may have affect on $\mathcal{R}_{0}$ and the total infectious. Besides, we complete the sensitivity analysis of the parameters with $\mathcal{R}_{0}$ and the total infectious (see Fig. 4, Table 5). When we take measures to control TB, the result is shown in Fig. 5. In general, we find we can control the factors with $\alpha, g, \psi, r$, $\beta_{i}, i=1,2,3, \phi$ and $\delta$. From the result in Fig. 5, we can find that $r$ has the greatest effect on the total infected, then $g$ has the second greatest effect, and others have a similar effect.

Strategy 1: We can find $r$ is strongly negatively correlated with $\mathcal{R}_{0}$, which means it is wise to use Chemoprophylaxis to control TB. With our control, the TB can be significantly controlled from Fig. 5. In order to prevent the TB outbreak, we can encourage people to have Chemoprophylaxis by the media, research on new and more efficient chemoprophylaxis to improve the effect, reduce the harm to the human body, and improve the therapeutic effect [72].

Strategy 2: The parameter $g$ has a negative effect on the $\mathcal{R}_{0}$, and with our control, it evidently reduced the total infectious. In doing so, we should take more money and energy to research on new medicine and therapy to reduce the period of treatment [73]. Nowadays, despite improvement in the treatment of TB, it remains the second leading cause of death in the world. Therefore, we still have a long way to eradicate TB.

Strategy 3: From Fig. 4, we can see $\beta_{i}, i=1,2,3$ have a positive effect on $\mathcal{R}_{0}$. With our control, we can greatly reduce the total infectious by reducing the transmission rate. Although we have placed emphasis on treating TB patients in isolation the majority were infected TB through person-to-person contact each year [74]. We must strictly monitor, take protective measures to avoid $\mathrm{TB}$, and examine outsiders to prevent contact with $\mathrm{TB}$ patients [75]. If we decrease half of $\beta_{i}, i=1,2,3$, we can find the total infectious will drop (see Fig. 5).

Strategy 4: It is also clearly shown that $\psi$ is positively correlated with $\mathcal{R}_{0}$ and the total infectious, which means the longer the vaccine lasts, the easier it is to control TB. We make $0.8 \times \psi$, and find the total number of the infectious being reduced greatly, which means we can lower it to prevent TB. In addition to that, we can delay the duration of the BCG by researching on new and better vaccination to prevent infecting TB. Currently, one of the primary health interventions that can be used to prevent $\mathrm{TB}$ is to vaccinate children.
Strategy 5: The parameter $\delta$ exerts a positive effect on the $\mathcal{R}_{0}$, and $\alpha$ has a negative effect on the $\mathcal{R}_{0}$. Firstly, we can encourage people to complete the treatment to be fully cured by reducing the cost [76]. The state can make more medical insurance to relieve the financial burden an $\mathrm{d}$ help people heal. Secondly, we can educate people to learn more about TB treatment, to follow the doctor's plan, and to realize that health is more important than everything.

Strategy 6: It shows that $\phi$ is negatively correlated with both $\mathcal{R}_{0}$ and the total infectious, which means increasing the number of vaccinated people per year can protect people from contracting TB, and thus control TB. Even for the state with little burden of TB, BCG can help prevent TB.

In this paper, the data used for fitting is annual data. Due to the extensive time scale, the accuracy of the model will be reduced. In later research, we will choose monthly data. Although our model take into account factors such as slow-fast process [41-43], vaccination [24-26], reinfection [21-23], reactivated [29,30] and undiagnosed infection [21, 44], these factors are not enough. We did not take into account factors such as interactions with HIV $[27,28]$, immigration $[77,78]$ and drug-resistant TB bacilli $[69,79,80]$. In addition to the aforementioned factors, we also did not consider residents' medical expenditure and awareness of disease control to discuss the prevention and control measures in this study. As for the deficiencies in the study, we will analyze and discuss them in the follow-up study.

\section{Conclusion}

In general, from the analysis, it is evident that, similar to TB studies elsewhere, the prevalence of TB in the United States is heavily influenced by exposure, vaccination, and treatment effectiveness. In our study, we also found that chemoprophylaxis affects the prevalence of TB more than other factors. However, each coin has two sides. Chemoprophylaxis has certain harm to the human body [72], so we should research new and better measures to prevent and control TB. Based on the analysis, we propose some strategies to control and eliminate TB in two ways: prevention and treatment. Chemoprophylaxis stands out as the one that can greatly control TB with some side effects. Accordingly, we should do much research to find better Chemoprophylaxis.

In fact, because the recovered infectious may relapse, the difficulty of diagnosing and treating $\mathrm{TB}$ is hard to control. When all the control measures are implemented together (see Fig. 5, solid black line), the basic reproduction number of the model (1) is $0.6915<1$. However, the case does not disappear. Instead, the system stabilizes to the endemic equilibrium. The measures we propose may not eliminate $\mathrm{TB}$, but they are critically useful for controlling the epidemic of TB. According to the latest report, in the announcement came at the first WHO 


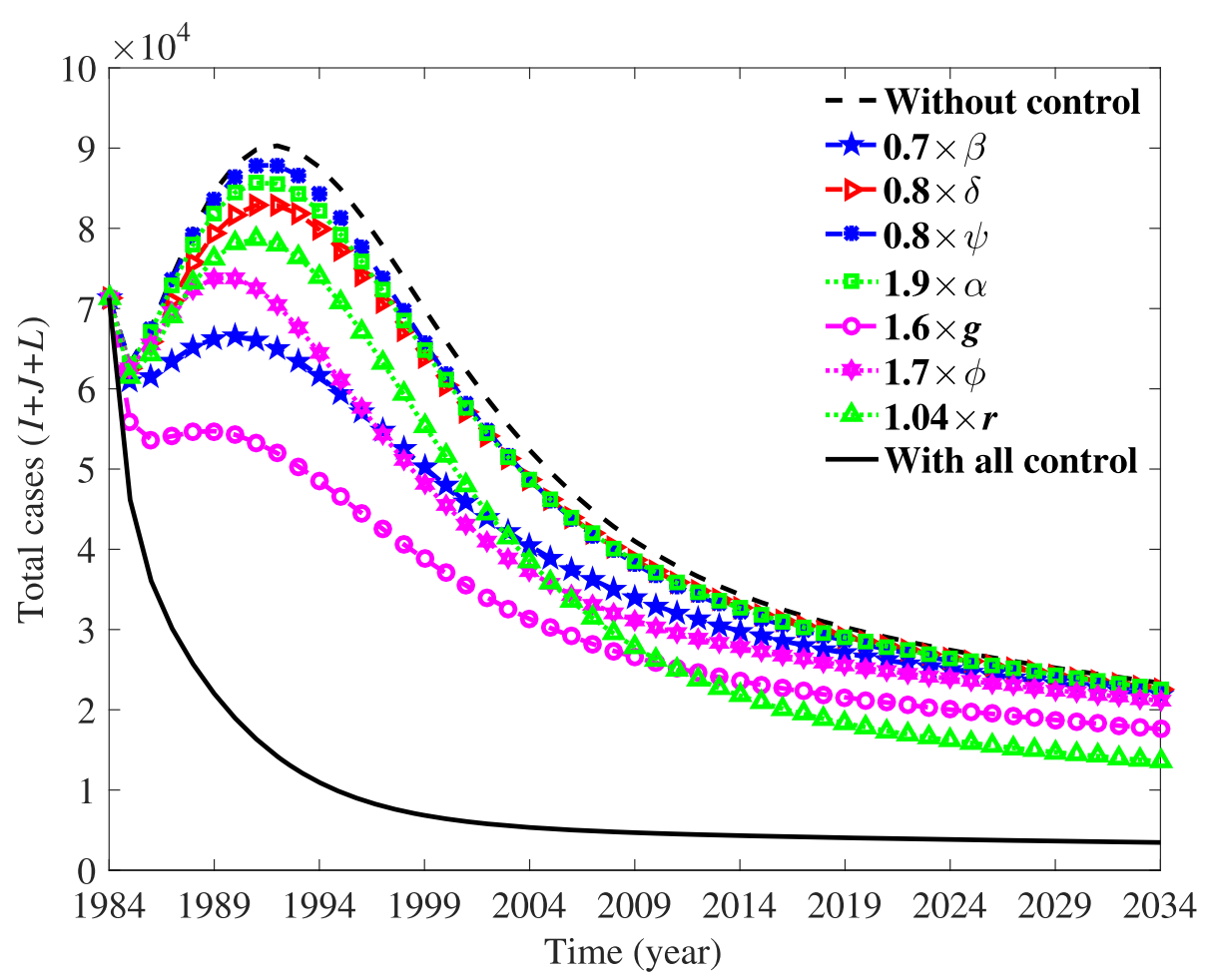

Fig. 5 Simulation of the total infectious with parameters $1.03 \times r=0.9496,1.5 \times \alpha=0.1503,1.5 \times g=0.7533,1.5 \times \phi=0.0750,0.9 \times \delta=0.5128$, $0.99 \times \psi=0.0505,0.7 \times \beta_{1}=3.1067,0.7 \times \beta_{2}=0.2096$ and $0.7 \times \beta_{3}=4.1063$, when one parameter takes a specific value, others take the value of the first column in Table 3. Differently, we synthesize the effects of three contact rates $\beta_{i,}, i=1,2,3$ into one contact rate $\beta$ effect. 'With all control' means that we let all parameters specific values simultaneously. 'Without control' is the situation which we take no measures. We can find $\psi$ has a mild effect on the total infected, with its line overlapping with 'without control' approximately

Global Ministerial Conference on Ending Tuberculosis in the Sustainable Development Era, 75 ministers agreed to take urgent measures to end TB by 2030 [81]. From our analysis, it is difficult to end TB until 2030 under the existing conditions (Fig. 5). Thus, we should find more and better methods to eradicate TB. Although it will be difficult to eliminate TB in a short period of time, we believe that in the future, with advanced technologies, TB can be eliminated.

\section{Appendix}

It is easy to see that the model (1) always has a disease-free equilibrium $P_{0}$, and the disease-free equilibrium, which are the solutions of the algebraic equations:

$$
\left\{\begin{array}{l}
\Lambda \chi+\varphi S-(\phi+\mu) V-\varepsilon v V=0, \\
\Lambda(1-\chi)+\phi V+\left(1-q_{1}-q_{2}\right) \gamma R-\varphi S-v S-\mu S=0, \\
\varepsilon v V+\left(1-p_{1}-p_{2}\right) v S+q_{2} \gamma R+\rho J-(1-r) k E-\mu E=0, \\
p_{1} v S+h(1-r) k E+\theta J+q_{1} \gamma R+\alpha L-(\delta+g) I-\left(\mu+d_{1}\right) I=0, \\
(1-h) k(1-r) E+p_{2} v S-(\theta+\rho) J-\left(\mu+d_{2}\right) J=0, \\
\delta I-(\alpha+\omega) L-\left(\mu+d_{3}\right) L=0, \\
g I+\omega L-(\gamma+\mu) R=0 \\
I=0 .
\end{array}\right.
$$

The disease-free equilibrium $P_{0}$ :

$$
\begin{aligned}
& P_{0}=\left((\varphi \Lambda+\mu \chi \Lambda) /\left(\mu \varphi+\mu \phi+\mu^{2}\right)\right. \\
& \left.\quad(\mu \Lambda+\phi \Lambda-\mu \chi \Lambda) /\left(\mu \varphi+\mu \phi+\mu^{2}\right), 0,0,0,0,0\right)
\end{aligned}
$$

The next-generation matrix approach is applied to calculate the basic reproduction number $\mathcal{R}_{0}$ [49]. For this purpose, we set $X=(V, S, E, I, J, L, R)$, there are $\dot{X}=$ $F-Q$, where:

$F=\left(0,0,\left(1-p_{1}-p_{2}\right) v S+\varepsilon v V, p_{1} v S, p_{2} v S, 0,0\right)^{T}$

and

$$
Q=\left(\begin{array}{c}
-(\Lambda \chi+\varphi S-(\phi+\mu) V-\varepsilon \nu V) \\
-\left(\Lambda(1-\chi)+\phi V+\left(1-q_{1}-q_{2}\right) \gamma R-\varphi S-\nu S-\mu S\right) \\
-\left(q_{2} \gamma R+\rho J-(1-r) k E-\mu E\right) \\
-\left(h(1-r) k E+\theta J+q_{1} \gamma R+\alpha L-(\delta+g) I-\left(\mu+d_{1}\right) I\right) \\
-\left((1-h) k(1-r) E-(\theta+\rho) J-\left(\mu+d_{2}\right) J\right) \\
-\left(\delta I-(\alpha+\omega) L-\left(\mu+d_{3}\right) L\right) \\
-(g I+\omega L-(\gamma+\mu) R)
\end{array}\right)
$$


Jacobian matrices $\mathcal{F}$ and $\mathcal{Q}$ at the disease-free equilibrium of $F$ and $Q$ are calculated respectively:

$$
\begin{aligned}
\mathcal{F} & =\left.\frac{\partial F(V, S, E, I, J, L, R)}{\partial(V, S, E, I, J, L, R)}\right|_{P_{0}} \\
& =\left[\begin{array}{ccccccc}
0 & 0 & 0 & 0 & 0 & 0 & 0 \\
0 & 0 & 0 & 0 & 0 & 0 & 0 \\
F_{1} & F_{2} & F_{3} & F_{4} & F_{5} & F_{6} & F_{3} \\
W_{1} & p_{1} A_{1}+W_{1} & W_{1} & \frac{S \beta_{1} p_{1}}{N}+W_{1} & \frac{S \beta_{2} p_{1}}{N}+W_{1} & \frac{S \beta_{3} p_{1}}{N}+W_{1} & W_{1} \\
W_{2} & p_{2} A_{1}+W_{2} & W_{2} & \frac{S \beta_{1} p_{2}}{N}+W_{2} & \frac{S \beta_{2} p_{2}}{N}+W_{2} & \frac{S \beta_{3} p_{2}}{N}+W_{2} & W_{2} \\
0 & 0 & 0 & 0 & 0 & 0 & 0 \\
0 & 0 & 0 & 0 & 0 & 0 & 0
\end{array}\right]
\end{aligned}
$$

where:

$$
\begin{aligned}
& F_{1}=\varepsilon A_{1}+S\left(p_{1}+p_{2}-1\right) A_{2}-V \varepsilon A_{2} \\
& F_{2}=S\left(p_{1}+p_{2}-1\right) A_{2}-V \varepsilon A_{2}-\left(p_{1}+p_{2}-1\right) A_{1} \\
& F_{3}=S\left(p_{1}+p_{2}-1\right) A_{2}-V \varepsilon A_{2} \\
& F_{4}=S\left(p_{1}+p_{2}-1\right) A_{2}-V \varepsilon A_{2}-\frac{S \beta_{1}\left(p_{1}+p_{2}-1\right)+V \beta_{1} \varepsilon}{N} \\
& F_{5}=S\left(p_{1}+p_{2}-1\right) A_{2}-V \varepsilon A_{2}-\frac{S \beta_{2}\left(p_{1}+p_{2}-1\right)+V \beta_{2} \varepsilon}{N} \\
& F_{6}=S\left(p_{1}+p_{2}-1\right) A_{2}-V \varepsilon A_{2}-\frac{S \beta_{3}\left(p_{1}+p_{2}-1\right)+V \beta_{3} \varepsilon}{N}
\end{aligned}
$$

and

$$
\begin{aligned}
& W_{1}=-S p_{1} A_{2} \\
& W_{2}=-S p_{2} A_{2} \\
& A_{1}=\frac{I \beta_{1}+J \beta_{2}+L \beta_{3}}{N} \\
& A_{2}=\frac{I \beta_{1}+J \beta_{2}+L \beta_{3}}{N^{2}} \\
& N=V+S+E+I+L+J+R
\end{aligned}
$$$$
\mathcal{Q}=\left.\frac{\partial Q(V, S, E, I, J, L, R)}{\partial(V, S, E, I, J, L, R)}\right|_{P_{0}}
$$$$
=\left[\begin{array}{ccccccc}
Q_{1} & -\phi-M & -M & \frac{V \beta_{1} \varepsilon}{N}-M & \frac{V \beta_{2} \varepsilon}{N}-M & \frac{V \beta_{3} \varepsilon}{N}-M & -M \\
-\psi-S A_{2} & Q_{2} & -S A_{2} & \frac{S \beta_{1}}{N}-S A_{2} & \frac{S \beta_{2}}{N}-S A_{2} & \frac{S \beta_{3}}{N}-S A_{2} & Q_{8} \\
0 & 0 & Q_{3} & 0 & \rho & 0 & -\gamma q_{2} \\
0 & 0 & h k(r-1) & Q_{5} & -\theta & -\alpha & -\gamma q_{1} \\
0 & 0 & Q_{4} & 0 & Q_{6} & 0 & 0 \\
0 & 0 & 0 & -\delta & 0 & Q_{7} & 0 \\
0 & 0 & 0 & -g-\omega & 0 & 0 & \gamma+\mu
\end{array}\right]
$$

where

$$
\begin{aligned}
& M=V \varepsilon A_{2} \\
& Q_{1}=\mu+\psi+\varepsilon A_{1}-M \\
& Q_{2}=\mu+\phi+A_{1}-S A_{2} \\
& Q_{3}=\mu-k(r-1) \\
& Q_{4}=-k(h-1)(r-1) \\
& Q_{5}=d_{1}+\delta+g+\mu \\
& Q_{6}=d_{2}+\mu+\rho+\theta \\
& Q_{7}=\alpha+d_{3}+\mu+\omega \\
& Q_{8}=\gamma\left(q_{1}+q_{2}-1\right)-S A_{2}
\end{aligned}
$$

then, one can obtain $G=\mathcal{F} \mathcal{Q}^{-1}$. The basic reproduction number $R_{0}=\max \left\{\lambda_{\mathcal{R}_{0}}\right\}$, where $\lambda_{\mathcal{R}_{0}}$ are the eigenvalues of $G$. Because $\mathcal{R}_{0}$ is complex, we don't give the detailed expression, and compute it via Matlab (the Mathworks, Inc.).

\section{Abbreviations}

TB: Tuberculosis; DESQP: global differential evolution and local sequential quadratic programming; LHS: Latin hypercube sampling; PRCC: partial rank correlation coefficients; ODE: ordinary differential equations; MAPE: the mean absolute percentage error; RMSPE: the root mean square percentage error; AIDS: acquired immunodeficiency syndrome; HIV: human immunodeficiency virus; MTB: Mycobacterium tuberculosis; BCG: Bacillus Calmette - Guerin; China's CDC: Chinese Center for Disease Control and Prevention; WHO: The World Health Organization; SQP: sequence quadratic program; SA: sensitivity analysis; UA: uncertain sensitivity analysis; TST: tuberculin skin test; Th1:

T-helper 1

\section{Acknowledgments}

We would like to thank anonymous reviewers for constructive suggestions, which significantly improved this manuscript.

\section{Authors' contributions}

WY and LY conceptualized and designed the study, drafted the initial manuscript, and approved the final manuscript as submitted. WY analyzed the data and simulated parameters. HM, WX, JL and YY carried out the initial analyses, reviewed and revised the manuscript. All authors read and approved the final manuscript.

\section{Funding}

The work was partially supported by the National Natural Science Foundation of China (No. 11901059), Natural Science Foundation of Hubei Province, China (No. 2019CFB353) and Undergraduate Training Program of Yangtze University for Innovation and Entrepreneurship (No. 2017036). The funding body had no role in the study design, collection, analysis, interpretation of data, and writing the manuscript.

\section{Availability of data and materials}

The data that support the findings of this study are available from the Center for Disease Control and Prevention (CDC)

(https://www.cdc.gov/tb/statistics/reports/2017/table1.htm), these network direct data are completely open, and we count these data.

\section{Ethics approval and consent to participate}

Since no individual patient's data was collected, the ethical approval or individual consent was not applicable.

\section{Consent for publication}

Not applicable.

\section{Competing interests}

The authors declare that there is no conflict of interests regarding the publication of this article. No authors have potential conflicts of interest with reference to this work.

\section{Author details}

${ }^{1}$ School of Information and Mathematics, Yangtze University, Jingzhou 434023, China, Nanhuan Road, 434023 Jingzhou, China. ${ }^{2}$ Institute of Applied Mathematics, Yangtze University, Nanhuan Road, 434023 Jingzhou, China.

${ }^{3}$ Department of Respiratory Medicine, Jingzhou Hospital of Traditional Chinese Medicine, Jiangjin East Road, 434000 Jingzhou, China. ${ }^{4}$ Laboratory Department, Jingzhou Maternal and Child Health Hospital, Jingzhong Road, 434000 Jingzhou, China.

Received: 17 November 2019 Accepted: 14 July 2020

Published online: 28 July 2020

\section{References}

1. Moualeu-Ngangue D, Röblitz S, Ehrig R, Deuflhard P. Parameter identification in a tuberculosis model for Cameroon. PloS one. 2015;10(4): 0120607.

2. World Health Organization (WHO). Global Tuberculosis Report 2018 WHO: World Health Organization; 2018. https://apps.who.int/iris/handle/ 10665/274453. Accessed 10 July 2020.

3. Centers for Disease Control and Prevention. How TB Spreads. https:// www.cdc.gov/tb/topic/basics/howtbspreads.htm. Accessed 11 Mar 2016.

4. Castillo-Chavez C, Song B. Dynamical models of tuberculosis and their applications. Math Biosci Eng. 2004;1 (2):361-404.

5. World Health Organization (WHO). BCG vaccines: WHO position paperFebruary 2018. Releve Epidemiologique Hebdomadaire. 2018;93(8):73-96. 
6. Centers for Disease Control and Prevention (CDC). Tuberculosis (TB), Reported Tuberculosis in the United States, 2018. https://www.cdc.gov/ tb/statistics/reports/2018/table1.htm. Accessed 6 Sept 2019.

7. Quick Easy Money. PopulationStat-world statistical data: United States Population. https://populationstat.com/united-states/. Accessed 7 June 2020

8. Aparicio J, Capurro A, Castillo-Chavez C. Long-term dynamics and re-emergence of tuberculosis. Inst Math Appl. 2002;125:351.

9. Centers for Disease Control and Prevention (CDC). Tuberculosis (TB), Reported Tuberculosis in the United States, 2018. Tuberculosis Cases and Percentages, by Reason Therapy Was Stopped Reporting Areas, 2016 https://www.cdc.gov/tb/statistics/reports/2018/table49.htm. Accessed 6 Sept 2019.

10. Centers for Disease Control and Prevention (CDC). Tuberculosis (TB), Reported Tuberculosis in the United States, 2018. Tuberculosis Cases and Percentages, by Reason Tuberculosis Therapy Was Stopped: United States, 1993-2016. https://www.cdc.gov/tb/statistics/reports/2018/ table12.htm. Accessed 6 Sept 2019.

11. Castillo-Chavez C, Feng Z. To treat or not to treat: the case of tuberculosis. J Math Biol. 1997;35(6):629-56.

12. Kolata G. First documented case of TB passed on airliner is reported by the US. New York Times. 1995;3:222-7.

13. World Health Organization, et al. Weekly epidemiological record. 2014, vol. 89, 43. Weekly Epidemiological Record= Relevé épidémiologique hebdomadaire. 2014;89(43):465-92.

14. Roy A, Eisenhut M, Harris R, Rodrigues L, Sridhar S, Habermann S, Snell $L$, Mangtani $P$, Adetifa I, Lalvani A, et al. Effect of bcg vaccination against mycobacterium tuberculosis infection in children: systematic review and meta-analysis. BMJ. 2014;349:4643.

15. Fjallbrant $H$, Ridell $M$, Larsson $L$. Primary vaccination and revaccination of young adults with BCG: A study using immunological markers. Scand J Infect Dis. 2007;39(9):792-8.

16. Centers for Disease Control and Prevention (CDC). Tuberculosis (TB), Basic TB Facts: Vaccines. https://www.cdc.gov/tb/topic/basics/vaccines.htm. Accessed 15 Mar 2016.

17. Listed $\mathrm{N}$. The role of BCG vaccine in the prevention and control of tuberculosis in the United States. A joint statement by the Advisory Council for the Elimination of Tuberculosis and the Advisory Committee on Immunization Practices. Mmwr Recomm Rep. 1996;45(RR-4):1-18. https://www.cdc.gov/mmwr/preview/mmwrhtml/00041047.htm. Accessed 26 Apr 1996.

18. Luca S, Mihaescu T. History of BCG vaccine. Maedica. 2013;8(1):53-8.

19. Bhunu C, Garira W, Mukandavire Z, Zimba M. Tuberculosis transmission model with chemoprophylaxis and treatment. Bull Math Biol. 2008;70(4): 1163-91.

20. Waaler H, Geser A, Andersen S. The use of mathematical models in the study of the epidemiology of tuberculosis. Am J Public Health Nations Health. 1962;52(6):1002-13.

21. Moualeu D, Yakam A, Bowong S, Temgoua A. Analysis of a tuberculosis model with undetected and lost-sight cases. Commun Nonlinear Sci Numer Simul. 2016;41:48-63.

22. Rodrigues P, Gomes M, Rebelo C. Drug resistance in tuberculosis-a reinfection model. Theor Popul Biol. 2007;71 (2):196-212.

23. Feng Z, Castillochavez C, Capurro A. A model for tuberculosis with exogenous reinfection. Theor Popul Biol. 2000;57(3):235-47.

24. Liu J, Zhang T. Global stability for a tuberculosis model. Math Comput Model. 2011;54(1-2):836-45.

25. Bhunu C, Garira W, Mukandavire Z, Magombedze G. Modelling the effects of pre-exposure and post-exposure vaccines in tuberculosis control. J Theor Biol. 2008;254(3):633-49.

26. Mantillabeniers N, Gomes M. Mycobacterial ecology as a modulator of tuberculosis vaccine success. Theor Popul Biol. 2009;75(2):142-52.

27. Narendran G, Swaminathan S. TB-HIV co-infection: a catastrophic comradeship. Oral Dis. 2016;22:46-52.

28. Centers for Disease Control and Prevention (CDC). Tuberculosis (TB), TB and HIV Coinfection. https://www.cdc.gov/tb/topic/basics/ tbhivcoinfection.htm. Accessed 12 Mar 2016.

29. Okuonghae D, Omosigho $S$. Analysis of a mathematical model for tuberculosis: What could be done to increase case detection. J Theor Biol. 2011;269(1):31-45.
30. Basu S, Andrews J, Poolman E, Gandhi N, Shah N, Moll A, Moodley P, Galvani A, Friedland G. Prevention of nosocomial transmission of extensively drug-resistant tuberculosis in rural South African district hospitals: an epidemiological modelling study. The Lancet. 2007;370(9597):1500-7.

31. Choi S, Jung E. Optimal tuberculosis prevention and control strategy from a mathematical model based on real data. Bull Math Biol. 2014;76(7): 1566-89.

32. Murray CJ, Salomon JA. Modeling the impact of global tuberculosis control strategies. Proceedings of the National Academy of Sciences. 1998;95(23):13881-6.

33. Roeger LIW, Feng Z, Castillo-Chavez C. Modeling TB and HIV co-infections. Math Biosci Eng. 2009;6(4):815.

34. Bowong S, Tewa J. Global analysis of a dynamical model for transmission of tuberculosis with a general contact rate. Commun Nonlinear Sci Numer Simul. 2010;15(11):3621-31.

35. Revelle C, Lynn W, Feldmann F. Mathematical models for the economic allocation of tuberculosis control activities in developing nations. Am Rev Respir Dis. 1967;96(5):893-909.

36. Buonomo B, dOnofrio A, Lacitignola D. Global stability of an SIR epidemic model with information dependent vaccination. Math Biosci. 2008;216(1):9-16.

37. Whang $S$, Choi S, Jung E. A dynamic model for tuberculosis transmission and optimal treatment strategies in South Korea. J Theor Biol. 2011;279(1): $120-31$.

38. Mishra B, Srivastava J. Mathematical model on pulmonary and multidrug-resistant tuberculosis patients with vaccination. J Egypt Math Soc. 2014;22(2):311-6.

39. Gao D, Huang N. Optimal control analysis of a tuberculosis model. Appl Math Model. 2018;58:47-64.

40. Center for Health Market Innovations. Global Tuberculosis Report; 2012. https://healthmarketinnovations.org/document/global-tuberculosisreport-2012. Accessed 23 July 2013.

41. Yang Y, Li J, Ma Z, Liu L. Global stability of two models with incomplete treatment for tuberculosis. Chaos Solitons Fractals. 2010;43(1):79-85.

42. Bowong S, Tewa J. Mathematical analysis of a tuberculosis model with differential infectivity. Commun Nonlinear Sci Numer Simul. 2009;14(11): 4010-21.

43. Aparicio JP, Capurro AF, Castillochavez C. Markers of Disease Evolution: The Case of Tuberculosis. J Theor Biol. 2002;215(2):227-37.

44. Zhang J, Li Y, Zhang X. Mathematical modeling of tuberculosis data of China. J Theor Biol. 2015;365:159-63.

45. Moualeu D, Weiser M, Ehrig R, Deuflhard P. Optimal control for a tuberculosis model with undetected cases in Cameroon. Commun Nonlinear Sci Numer Simul. 2015;20(3):986-1003.

46. Storn R, Price K. Differential evolution-a simple and efficient heuristic for global optimization over continuous spaces. J Glob Optim. 1997;1 1(4): 341-59.

47. Sivasubramani S, Swarup K. Hybrid DE-SQP algorithm for non-convex short term hydrothermal scheduling problem. Energy Convers Manag. 2011;52(1):757-61.

48. Li Y, Wang L, Pang L, Liu S. The data fitting and optimal control of a hand, foot and mouth disease (HFMD) model with stage structure. Appl Math Comput. 2016;276:61-74.

49. Van den Driessche P., Watmough J. Reproduction numbers and sub-threshold endemic equilibria for compartmental models of disease transmission. Math Biosci. 2002;180((1-2)):29-48.

50. Wang L, Li Y, Pang L. Dynamics analysis of an epidemiological model with media impact and two delays. Math Probl Eng. 20162016.

51. Chen X, Yao W, Zhao Y, Chen X, Zhang J, Luo Y. The hybrid algorithms based on differential evolution for satellite layout optimization design. In: 2018 IEEE Congress on Evolutionary Computation (CEC). IEEE; 2018. p. 1-8.

52. Attaviriyanupap $P$, Kita H, Tanaka E, Hasegawa J. A hybrid EP and SQP for dynamic economic dispatch with nonsmooth fuel cost function. IEEE Trans Power Syst. 2002;17(2):411-6.

53. Victoire T, Jeyakumar A. Hybrid PSO-SQP for economic dispatch with valve-point effect. Electr Power Syst Res. 2004;71(1):51-9.

54. Aandahl R, Stadler T, Sisson S, Tanaka M. Exact vs. approximate computation: Reconciling different estimates of mycobacterium tuberculosis epidemiological parameters. Genetics. 2014;196(4):1227-30. 
55. Tanaka M, Francis A, Luciani F, Sisson S. Using approximate bayesian computation to estimate tuberculosis transmission parameters from genotype data. Genetics. 2006;173(3):1511-20.

56. Stadler T. Inferring epidemiological parameters on the basis of allele frequencies. Genetics. 2011;188(3):663-72.

57. Lou P, Wang L, Zhang X, Xu J, Wang K. Modelling seasonal brucellosis epidemics in bayingolin mongol autonomous prefecture of Xinjiang, China, 2010-2014. BioMed Res Int. 20162016

58. Zhang T, Wang K, Zhang X. Modeling and analyzing the transmission dynamics of HBV epidemic in Xinjiang, China. PloS one. 2015;10(9): 0138765.

59. Zheng Y, Rundell A. Comparative study of parameter sensitivity analyses of the TCR-activated Erk-MAPK signalling pathway. IEE Proc Syst Biol. 2006;153(4):201-11.

60. Xiao Y, Tang S, Zhou Y, Smith R, Wu J, Wang N. Predicting the HIV/AIDS epidemic and measuring the effect of mobility in mainland China. J Theor Biol. 2013;317:271-85

61. Marino S, Hogue I, Ray C, Kirschner D. A methodology for performing global uncertainty and sensitivity analysis in systems biology. J Theor Biol. 2008;254(1):178-96.

62. Sumner T, Shephard E, Bogle I. A methodology for global-sensitivity analysis of time-dependent outputs in systems biology modelling. J R Soc Interface. 2012;9(74):2156-66.

63. Ihekwaba A, Broomhead D, Grimley R, Benson N, Kell D. Sensitivity analysis of parameters controlling oscillatory signalling in the NF- $\kappa \mathrm{B}$ pathway: the roles of IKK and $\mid \kappa B \alpha$. Syst Biol. 2004;1(1):93-103.

64. Hu D, Yuan J-M. Time-dependent sensitivity analysis of biological networks: coupled MAPK and PI3K signal transduction pathways. J Phys Chem A. 2006;110(16):5361-70.

65. World Health Organization (WHO). United States of America. https:// www.who.int/countries/usa/en/. Accessed 7 June 2020.

66. Borgdorff M, Sebek M, Geskus R, Kremer K, Kalisvaart N, van Soolingen D. The incubation period distribution of tuberculosis estimated with a molecular epidemiological approach. Int J Epidemiol. 2011;40(4):964-70.

67. Wikipedia. Tuberculosis. 2020. https://en.wikipedia.org/w/index.php? title=Tuberculosis\&oldid=961034230. Accessed 7 June 2020.

68. Lawn S, Zumla A. Tuberculosis. Lancet. 2011;378(9785):57.

69. Dye C, Williams B. Criteria for the control of drug-resistant tuberculosis. Proc Natl Acad Sci U S A. 2000;97(14):8180-5.

70. Dye C, Scheele S, Dolin P, Pathania V, Raviglione M. Global burden of tuberculosis: Estimated incidence, prevalence, and mortality by country. JAMA. 1999;282(7):677-86.

71. Bacaër N, Ouifki R, Pretorius C, Wood R, Williams B. Modeling the joint epidemics of TB and HIV in a South African township. J Math Biol. 2008;57(4):557.

72. Pannucci C, Swistun L, MacDonald J, Henke P, Brooke B. Individualized venous thromboembolism risk stratification using the 2005 Caprini score to identify the benefits and harms of chemoprophylaxis in surgical patients: a meta-analysis. Ann Surg. 2017;265(6):1094-103.

73. Koul A, Arnoult E, Lounis N, Guillemont J, Andries K. The challenge of new drug discovery for tuberculosis. Nature. 2011;469(7331):483.

74. Gammaitoni L, Nucci M. Using a mathematical model to evaluate the efficacy of TB control measures,. Emerg Infect Dis. 1997;3(3):335.

75. Feng Z, Huang W, Castillo-Chavez C. On the role of variable latent periods in mathematical models for tuberculosis. J Dyn Diff Equat. 2001;13(2):425-52.

76. Gebremariam M, Bjune G, Frich J. Barriers and facilitators of adherence to TB treatment in patients on concomitant TB and HIV treatment: $\mathrm{a}$ qualitative study. BMC Public Health. 2010;10(1):651.

77. Jia Z, Tang G, Jin Z, Dye C, Vlas S, Li X, Feng D, Fang L, Zhao W, Cao W. Modeling the impact of immigration on the epidemiology of tuberculosis. Theor Popul Biol. 2008;73(3):437-48.

78. Liu L, Ren X, Jin Z. Threshold dynamical analysis on a class of age-structured tuberculosis model with immigration of population. Adv Differ Equ. 2017;2017(1):1-21.

79. Trauer J, Denholm J, Mcbryde E. Construction of a mathematical model for tuberculosis transmission in highly endemic regions of the Asia-pacific. J Theor Biol. 2014;358:74-84.

80. Zhao Y, Xu S, Wang L, Chin D, Wang S, Jiang G, Xia H, Zhou Y, Li Q, Ou $X$, et al. National Survey of Drug-Resistant Tuberculosis in China. New Engl J Med. 2012;366(23):2161-70.
81. World Health Organization (WHO). New global commitment to end tuberculosis. https://www.who.int/news-room/detail/17-11-2017-newglobal-commitment-to-end-tuberculosis. Accessed 17 Nov 2017.

\section{Publisher's Note}

Springer Nature remains neutral with regard to jurisdictional claims in published maps and institutional affiliations.
Ready to submit your research? Choose BMC and benefit from:

- fast, convenient online submission

- thorough peer review by experienced researchers in your field

- rapid publication on acceptance

- support for research data, including large and complex data types

- gold Open Access which fosters wider collaboration and increased citations

- maximum visibility for your research: over $100 \mathrm{M}$ website views per year

At BMC, research is always in progress.

Learn more biomedcentral.com/submissions 